\title{
Optical fiber distributed sensing based on Rayleigh scatter frequency domain reflectometry for Sodium leakage detection within SFR reactors
}

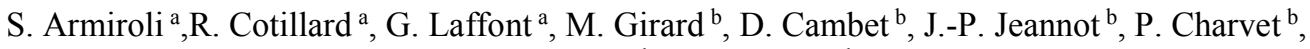 \\ S. Albaladéjo ${ }^{b}$ and S. Lusso ${ }^{b}$. \\ ${ }^{a}$ CEA, LIST, Laboratoire Capteurs et Architecture Electronique, 91191 Gif-sur-Yvette cedex, France. \\ ${ }^{\mathrm{b} C E A}$, DEN, DTN, 13108 Saint Paul lez Durance cedex, France.
}

\begin{abstract}
As part of the Generation IV international forum, CEA is performing research on Sodium-cooled fast neutron reactors. Liquid sodium holds many advantages such as its boiling point around $900^{\circ} \mathrm{C}$, its high thermal conductivity, its ability to solidify itself after cooling. Moreover Sodium is a weak absorber of neutrons.

Because of the sodium's high chemical reactivity, its use as coolant in nuclear reactor leads to new safety issues and technical challenges such as the development of efficient leak detection system adapted to pipelines or tanks. Laboratories of the French Alternative Energies and Atomic Energy Commission (LIST-LCAE, DEN-DTN) are working together to realize tests and studies about an innovative sodium leakage detection system involving optical fiber distributed temperature and strain sensing techniques.
\end{abstract}

In this paper, we propose to illustrate how optical fiber sensors are used to monitor liquid sodium leaks at the outer surface of a large diameter pipe. Single mode optical fibers are promising candidates for instrumentation within any nuclear facilities due to their low intrusivity, their electromagnetic immunity, their capability to withstand high temperatures ranges and their reliability even when operating in harsh and radiative environments. The optical fiber sensing technology used in this paper is the Optical Frequency Domain Reflectometry. The operating principle of the instrument is a frequency analysis of the Rayleigh backscattered light along the core of the optical fiber. Collecting and processing this backscattered signal, an OFDR trace is obtained which is similar to a fingerprint of the heterogeneities (refractive index variations or distortion of the fiber's geometry) distributed along the length of the optical fiber's core. The apparition of any spatially strain or temperature change along the fiber's core will locally modify the Rayleigh backscattered signal. One can then follow in quasi real time the temperature change or strain modifications over a typically 70 meter long predefined segment of the optical fiber sensor. The sensing optical fiber then operates as a distributed sensor. The measurement resolution of this technique is typically $1{ }^{\circ} \mathrm{C}$ and 10 micro-strains (similar to $0.001 \%$ of elongation) but specific temperature calibration has to be done for high temperature operating range. In this study, we are using a single mode optical fiber SMF1500[9/125], $9 \mu \mathrm{m}$ core diameter and $125 \mu \mathrm{m}$ cladding diameter similar to those used for telecommunication application. The optical fiber is protected using a metallic sheaths to avoid any damages by a direct contact with liquid sodium.

The leakage experiment is realized in the CEA $20 \mathrm{~m}$ longoptical fiber probe. The lagging of the mock-up is composed by several layers of thermal materials to confine the leakage on the main pipe and preserve the external environment from damages. The pipe of the mock-up can be maintained at high temperatures up to $550^{\circ} \mathrm{C}$ to simulate the liquid sodium temperature. The leakage is simulated by a secondary pipe placed inside the mock-up and its extremity leaves the main pipe on the top of it. The optical fiber is disposed directly on the main pipe and between the lagging's layers following a helical pattern. Any leak of Sodium is detected as a localized temperature change or "hot spot". We can then study the sodium leakage expansion around the pipe over time and estimate the seriousness of the leak thanks to alerts. In a near future, a 3D cartography will be proposed to display on real time the sodium expansion around the pipe.

Keywords: Optical Fiber Sensor, OFDR, Rayleigh backscattering, leakage detection, liquid sodium, fast reactor 